This item was submitted to Loughborough's Research Repository by the author.

Items in Figshare are protected by copyright, with all rights reserved, unless otherwise indicated.

\title{
Changes in core temperature during an elite female rugby sevens tournament
}

PLEASE CITE THE PUBLISHED VERSION

https://doi.org/10.1123/ijspp.2019-0375

PUBLISHER

Human Kinetics

VERSION

AM (Accepted Manuscript)

PUBLISHER STATEMENT

Accepted author manuscript version reprinted, by permission, from International Journal of Sports Physiology and Performance, 2020, 15 (4): 571-580, https://doi.org/10.1123/ijspp.2019-0375. (C Human Kinetics, Inc.

LICENCE

CC BY-NC-ND 4.0

\section{REPOSITORY RECORD}

Henderson, Mitchell, Bryna CR Chrismas, Christopher J Stevens, Aaron J Coutts, and Lee Taylor. 2020. "Changes in Core Temperature During an Elite Female Rugby Sevens Tournament". Loughborough University. https://hdl.handle.net/2134/13234898.v1. 
1 Title: Core temperature changes during an elite female rugby sevens tournament

Submission Type: Original Research

Authors: Mitchell J. Henderson ${ }^{1,2,3}$, Bryna C.R. Chrismas ${ }^{4}$, Christopher J. Stevens ${ }^{5,6}$, Aaron J. Coutts $^{1,3}$, Lee Taylor ${ }^{7,1,3,8^{*}}$

\section{Affiliations:}

${ }^{1}$ University of Technology Sydney (UTS), Sport \& Exercise Discipline Group, Faculty of Health, Australia.

${ }^{2}$ Australian Rugby Sevens, Rugby Australia (RA), Sydney, Australia.

${ }^{3}$ University of Technology Sydney (UTS), Human Performance Research Centre, Australia. ${ }^{4}$ Qatar University, Sport Science Program, College of Arts and Science, Doha, Qatar.

${ }^{5}$ Southern Cross University, School of Health and Human Sciences, Coffs Harbour, Australia. ${ }^{6}$ Southern Cross University, Centre for Athlete Development, Experience and Performance, Coffs Harbour, Australia.

${ }^{7}$ Loughborough University, School of Sport, Exercise and Health Sciences, Loughborough, UK.

${ }^{8}$ ASPETAR, Qatar Orthopaedic and Sports Medicine Hospital, Athlete Health and Performance Research Centre, Doha, Qatar.

\section{*Address for Correspondence}

Dr Lee Taylor, Loughborough University, School of Sport, Exercise and Health Sciences, Loughborough, Leicestershire, LE11 3TU, UK.

Email: 1.taylor2@lboro.ac.uk Phone:+44 1509226302

Preferred Running Head: Core temperature during elite female rugby sevens
Abstract word count:
212
Text-only word count: 3459 (excluding 63 words used for table/figure suggested location)
Number of figures: 4
Number of tables: 


\section{Abstract}

43 Purpose: Characterise player core temperature (Tc) across a World Rugby Women's Sevens 44 Series (WRWSS) tournament day and determine the efficacy of commonly employed cold water immersion (CWI) protocols. Methods: Tc was measured in twelve elite female rugby sevens athletes across 3 games (G1-3) from day 1 of the Sydney WRWSS tournament.

47 Exertional heat illness symptoms, perceptual scales, CWI details, playing minutes, external 48 load data (measured by Global Positioning Systems) and wet blub globe temperature (range: $49 \quad 18.5-20.1^{\circ} \mathrm{C}$ ) were also collected. Linear mixed models and magnitude-based inferences were 50 used to assess differences in Tc between periods [G1-3 and warm-ups (WU)]. Results: Average Tc was very likely lower (ES; $\pm 90 \% \mathrm{CL},-0.33 ; \pm 0.18)$ in G1 compared to G2. Peak Tc was very likely $(0.71 ; \pm 0.28)$ associated with increased playing time. CWI did not remove the accumulated Tc due to WU and match-play activity $\left(\sim 1-2^{\circ} \mathrm{C}\right.$ rise in Tc still present compared to Tc at WU onset for players $\geq 6$ min match-play). Conclusions: Elite female WRWSS athletes experienced high Tc during WU (Tc peak $37.9-39.0^{\circ} \mathrm{C}$ ) and matches (Tc peak $37.9-39.8^{\circ} \mathrm{C}$ ), a magnitude known to reduce intermittent high-intensity physical performance $\left(\geq 39^{\circ} \mathrm{C}\right)$. The CWI protocol resulted in players ( $\geq 6$ min match-play) with $\mathrm{a} \sim 1-$ $2{ }^{\circ} \mathrm{C}$ raised Tc compared to Tc at WU onset.

60 Keywords: heat, elite, telemetric, hyperthermia 
64 Rugby sevens is a modified shortened version of 15-a-side rugby union, where teams play with 7 players (5 reserves), across 7-minute halves, on a full-sized rugby-union pitch. The World Rugby Women's Sevens Series (WRWSS) is the premier international rugby sevens series/competition, currently with 6 tournaments per season (changing to 8 from 2019/2020). Tournaments are competed over $2-3$ day periods, with up to 3 matches per day and $\sim 3$ hours between matches. WRWSS match-play is characterised by frequent bouts of high-intensity activity and collisions, ${ }^{1}$ with speeds reported as $85.8 \pm 3.9 \mathrm{~m} \cdot \mathrm{min}^{-1}$ with $11 \pm 2.7 \%$ performed above $5 \mathrm{~m} \cdot \mathrm{s}^{-1} \cdot 2$

The WRWSS tournaments are contested in the southern and northern hemispheres resulting in variable environmental match-day conditions, where teams may travel from their domestic winter months to compete in summer months elsewhere, often facing extremes of heat and/or humidity. Match-play data from the male equivalent (reporting higher physical match intensities $^{2}$ ) of the WRWSS within temperate [13.8 - 22.3 wet bulb globe temperature (WBGT); London 2017] and warm (21.4 - 27.0 WBGT; Singapore 2017) WBGT environments, has demonstrated high core body temperatures $\left(\mathrm{Tc}\right.$; peak $\mathrm{Tc}$ of $39.9^{\circ} \mathrm{C}$ and $39.6^{\circ} \mathrm{C}$ in temperate and warm conditions, respectively $\left.{ }^{3}\right)$. This presents a challenge to practitioners and players, as a $\mathrm{Tc} \geq 39^{\circ} \mathrm{C}$ can impair intermittent/repeated sprint based

81 performance through: (i) reductions in arterial oxygen delivery to the working muscles, (ii) greater reliance on anaerobic energy provision and (iii) accelerated accumulation of $\mathrm{H}^{+} .4$

Physical and physiological differences in thermoregulation exist between genders (e.g. greater subcutaneous fat content, larger ratio of body surface to body mass, and smaller sweat response to heat load in females). ${ }^{5}$ Coupling this with the known anthropometrics of elite rugby sevens athletes (e.g. females are shorter, lighter with a higher fat content ${ }^{2}$ ), female players may be 
more susceptible to rapid rises in $\mathrm{Tc} \geq 39^{\circ} \mathrm{C}$ and subsequent performance decrements ${ }^{4}$ for the same work produced. Considering the high Tc from temperate/warm match-play in males ${ }^{3}$ capable of reducing physical performance ${ }^{4}$, other hotter tournaments of the WRWSS and Tokyo 2020 [expected to be the hottest modern Olympics to date $\left(\sim 30^{\circ} \mathrm{C}\right.$, with relative

91 humidity exceeding $75 \%$, 6 ] may challenge practitioners to manage player body temperatures to achieve optimal performance. This may be of greater importance for female athletes given the performance variation observed across the menstrual cycle in hot, humid conditions. ${ }^{8}$

94 Cold water immersion (CWI) is a commonly used acute post-game (N.B several games within WRWSS tournament day) recovery tool for rugby sevens athletes, proposed to control hyperthermia, reduce muscle inflammation and damage, and decrease muscle soreness. ${ }^{9}$

97 However, the efficacy of rugby sevens specific CWI protocols ${ }^{10}$, within a real-world elite tournament scenario, is not available [e.g. total heat storage as a consequence of each match plus associated warm-up (WU), and whether the CWI protocol actually removes this].

100 Telemetric capsules could wirelessly, and relatively non-invasively, ${ }^{11}$ acquire this within 101 WRWSS tournament day Tc characterisation and CWI efficacy data.

102 This study aims to characterise Tc values for elite female athletes within and between matches of a WRWSS tournament day, and the efficacy of post-game CWI protocols. It is hypothesized

104 that (i) WRWSS match play will result in Tc values $\geq 39^{\circ} \mathrm{C}$ and (ii) the commonly employed 105 post-game CWI protocol will not entirely remove body heat gained from the WU and match106 play.

107 Methods

108 Subjects $* * *$ Table 1 near here please *** 
109 Data were collected from a total of twelve seasonally heat-acclimatised female athletes (see

110 Table 1 for details) from a single 2018-19 WRWSS team based in Sydney, Australia, across

111 one tournament day. Written informed consent was provided, under ethical approval from the

112 Southern Cross University Human Research Ethics Committee (ECN-18-216) in the spirit of

113 the Helsinki Declaration.

114 Design

115 Data were collected across the first day (three games played, two wins, one loss) of the

116 Australian WRWSS tournament in Sydney, Australia (February 1-3, 2019). Players had been

117 in the same time zone $\geq 14$ days prior to the tournament, thus, circadian misalignment was not

118 a confounding influence. In line with elite team sport practice, menstrual cycle could not be 119 controlled.

\section{Methodology}

121 Players ingested an e-Celsius ${ }^{\mathrm{TM}}$ telemetric capsule (BodyCap, Caen, France) the night prior to

122 the tournament, and another upon waking on the first match day. Tc data was only included

123 within the statistical model when $\geq 5$ hours had elapsed post-ingestion, a criteria used

124 previously to ensure the capsule was in the lower intestine..$^{3,12,13}$ Tc was sampled at 30 second

125 intervals, with data downloaded at the end of the day via a wireless data receiver (e-Viewer,

126 BodyCap, Caen, France). Capsules were set-up, calibrated, and handled as extensively outlined 127 elsewhere. $^{3,12,14}$ The e-Celsius ${ }^{\mathrm{TM}}$ system has been shown valid and reliable for intermittent128 running exercise, ${ }^{14}$ has excellent validity (ICC 1.00), test-retest reliability (ICC 1.00) and

129 inertia in water bath experiments between $36^{\circ} \mathrm{C}$ and $44^{\circ} \mathrm{C},{ }^{11}$ and has been used previously 130 within elite rugby sevens matches. ${ }^{3,11}$ 
131 Playing minutes for individual athletes for each game were collected by the team's sports 132 scientist. Specific pre-defined time periods relative to Tc were employed within analyses 133 (specific timings provided in Table 2):

- Baseline $(B L): \quad 60$ minutes prior to prime.

- Activation / Primer: Time spent completing pre-warm up movement. Time spent with team undertaking team warm-up. Time spent in the game.

139 Signs and symptoms of exertional heat illnesses (EHI) were collected following each game

140 immediately prior to the CWI protocol, using a modified survey instrument. ${ }^{15}$ Specifically, the

141 athletes were asked in a yes/no manner if they had experienced (i) cramping; (ii) vomiting; (iii)

142 nausea; (iv) severe headache; (v) collapsing/fainting; or (vi) any other symptom that might

143 relate to heat illness. ${ }^{15} \mathrm{CWI}$ was provided for teams by the local organisers, with submersion

144 time of day (see Table 2) and duration (see results section) recorded (water temperature range:

$1456.1-7.3^{\circ} \mathrm{C}$ ). Players were submerged to hip height for the duration of their CWI intervention.

146 No other post-game cooling intervention methods were used.

147 WBGT (SD-2010, Reed Instruments, NC, USA) was obtained immediately prior to, during and 148 post WU (in the WU area outside of the stadium) and matches (5 $\mathrm{m}$ from the sideline). WBGT 149 values obtained within each pre-defined time period were averaged and are presented in Table 150 2. The day prior to the tournament within the stadium WBGT peak was $37.5^{\circ} \mathrm{C}$, with 151 expectations that tournament day one would see similar WBGT values, however, conditions 152 on the day were surprisingly mild $\left(\leq 20^{\circ} \mathrm{C}\right.$ WBGT; see Table 2$)$. 
154 Activity profiles during matches were measured using $10 \mathrm{~Hz}$ GPS devices (EVO, GPSports, 155 Canberra, Australia). These have shown good inter-unit reliability for distance (m) (CV: 0.2\% $156 \pm 1.5)$, average speed $\left(\mathrm{m} \cdot \mathrm{min}^{-1}\right)(\mathrm{CV}: 0.2 \% \pm 1.5)$, max velocity $\left(\mathrm{m} \cdot \mathrm{s}^{-1}\right)(\mathrm{CV}: 0.2 \% \pm 1.5)$, high157 speed running (distance covered $\left.>5 \mathrm{~m} \cdot \mathrm{s}^{-1}\right) \quad(\mathrm{CV}: \quad 0.5 \% \pm 1.5)$, and average 158 acceleration/deceleration $\left(\mathrm{m} \cdot \mathrm{s}^{-2}\right)(\mathrm{CV}: 1.2 \% \pm 1.5) .{ }^{16}$ Each unit was assigned to an individual 159 player and worn in a small pouch in their match jersey, positioning the unit between the scapula 160 blades of the player. Following each match, devices were downloaded using the manufacturer's 161 proprietary software (GPSports Console, GPSports, Canberra, Australia). Metrics exported

162 from the GPS data included match duration $(\mathrm{min})$, average speed $\left(\mathrm{m} \cdot \mathrm{min}^{-1}\right)$, high-speed 163 running per minute (HSR $\cdot \mathrm{min}^{-1}$; average distance covered at $>5 \mathrm{~m} \cdot \mathrm{s}^{-1}$ per minute), very high164 speed running per minute (VHSR $\cdot \mathrm{min}^{-1}$; average distance covered at $>6 \mathrm{~m} \cdot \mathrm{s}^{-1}$ per minute), and average acceleration/deceleration (Ave Acc/Dec; $\mathrm{m} \cdot \mathrm{s}^{-2}$ ).

166 Thermal sensation (TS) was measured using a 17-point category ratio scale (where $0=$ 167 'unbearably cold' and $8=$ 'unbearably hot'). ${ }^{17}$ Thermal comfort (TC) was measured using a 168 10-point category ratio scale (where $1=$ 'comfortable' and $10=+1$ above 'extremely uncomfortable'). ${ }^{18}$ Rating of perceived exertion (RPE) was measured using CR-10 category ratio scale $\left(\right.$ where $0=$ rest and $10=$ maximal). ${ }^{18}$

171 The TS, TC, RPE (all post-game), Tc and GPS measures were obtained as per Figure 1, by the 172 same practitioner using standardised language and procedures.

*** Figure 1 near here please $* * *$

\section{Statistical Analyses}

175 Statistical analyses were performed using the Statistical Package for the Social Sciences

176 (SPSS) version 25 (IBM, SPSS Inc, Chicago, IL, USA), and magnitude-based inferences 
177 (MBIs) customizable spreadsheets, using the raw data. ${ }^{19}$ Initially, descriptive statistics were 178 generated, and normality checked using quantile-quantile (Q-Q) plots. ${ }^{20}$ Descriptive statistics are reported as median and range (minimum - maximum) unless otherwise stated. GraphPad

180 Prism 8 (GraphPad Software, CA, USA) was used to create Figures $2-4$. Individual player Tc 181 was determined and averaged for each pre-defined time period, with peak Tc values for each 182 period also extracted. At each time period the individual player change (delta; $\Delta$ ) within period, and relative to baseline (BL) were calculated. Additionally, minimum Tc values from each WU

184 (WU 1, WU 2, WU 3) were subtracted from peak Tc values from each game (G1 - 3), to calculate total heat gained across each WU and game pair ( $\Delta \mathrm{Tc}$ combined). In order to examine the effectiveness of CWI in removing the total heat gained in $\Delta \mathrm{Tc}$ combined, the $\Delta \mathrm{Tc}$ combined value was subtracted from the $\Delta \mathrm{CWI}$, providing a $\Delta \Delta \mathrm{CWI}$ value.

Linear mixed models (LMM) were used to determine differences in: (i) Tc (peak, average, $\Delta$ period, $\Delta \mathrm{BL}, \Delta$ combined) across all periods, and between WU and games; (ii) RPE, TS, TC, minutes played, and all external load (GPS) variables between games; and (iii) $\Delta \Delta \mathrm{CWI}$

191 between each period (i.e. CWI 1, CWI 2, CWI 3). Specifically, fixed (i.e. period) and random

192 (i.e. participant) effects for the LMM were fit for each dependent variable, ${ }^{21}$ and a nested effect design was used, where player was nested in the period. This design estimates mean differences in individual Tc, and also models the between-player variability in Tc within each period. The most appropriate model was chosen using the smallest Hurvich and Tsai criterion (AICC) ${ }^{22}$ in accordance with the principle of parsimony. The least squares mean test provided pairwise comparisons between the fixed effect. Raw differences are reported as mean $(95 \%$ confidence intervals). Normality and homogeneity of variance of the residuals were checked using Q-Q plots, and scatter plots respectively, and deemed plausible in each instance. Furthermore, the relationship between minutes played and all external load (GPS) variables on peak Tc was assessed using LMM (random coefficient model). Peak Tc for all three games was included in 
the model as a dependent variable (outcome), and minutes played and all external load (GPS) variables (all three games combined) were separately entered as fixed effects (predictors).

204 Using a random intercept and slope design, player identification was nested within the predictors. All analyses were initially performed with all players, and subsequently with

206 players who had $\geq 6 \min (\sim 40 \%$ of game time $)$ match-play per game only. Perceptual and

207 external load (GPS) data was modelled only for those players with $\geq 6$ min match-play per game only. Cohen's $d$ effect sizes (ES), and 90\% confidence limits (CLs) were obtained using the MBI spreadsheets, and categorized using standardised thresholds of; $<0.2$ trivial, $0.21-$

211 results showed a significant $p$ value (significance was accepted as $p \leq 0.05$ ). Differences were

212 considered real if there was a $>75 \%$ likelihood of the observed effect exceeding the smallest worthwhile effect $(0.20 \mathrm{x}$ between subject $\mathrm{SD})$, using the following qualitative descriptions;

$21475-95 \%$ likely, $95-99.5 \%$ very likely, and $>99.5 \%$ most likely. ${ }^{19}$ Data is reported as ES; $215 \pm 90 \%$ CL.

\section{Results}

217 Raw data for Tc for all players, and perceptual and GPS data for players who had $\geq 6$ min 218 match-play are presented in Tables 2 and 3 respectively.

220 Core temperature across all periods: Peak Tc was $\geq 39^{\circ} \mathrm{C}$ for five players in game 1, and six players in games 2 and 3 (Figure 2). Peak and average Tc, when compared to BL, was higher throughout all periods for all players ( $\mathrm{p}<0.001 ; \mathrm{p}<0.001$ respectively) and in those who had

$223 \geq 6$ min match-play ( $<<0.001 ; \mathrm{p}<0.001$, respectively). Differences are reported in Table 4 224 and Figure 2. 
*** Table 4 near here please $* * *$

226

*** Figure 2 near here please $* * *$

227 Core temperature between WU and games (all players): Differences $(\mathrm{p}=0.04)$ in average Tc 228 was seen between games. Players very likely $(-0.33 ; \pm 0.18)$ had a lower average Tc in game 1 229 compared to game $2\left[-0.27\left(-0.09\right.\right.$ to $\left.\left.-0.49^{\circ} \mathrm{C}\right) ; \mathrm{p}=0.007\right]$ with no further Tc derived differences 230 evident between games $(\mathrm{p} \geq 0.24)$ or between WU $(\mathrm{p} \geq 0.13)$ across day, see Figure 2 .

231 Core temperature between $W U$ and games (only players $>6$ min): Differences $(p=0.04)$ in $232 \Delta \mathrm{Tc}$ within period between games were evident. Players most likely $(0.91 ; \pm 0.31)$ had a greater $233 \Delta \mathrm{Tc}$ in game 1 compared to game $2\left[0.39\left(0.14\right.\right.$ to $\left.\left.0.63^{\circ} \mathrm{C}\right) ; \mathrm{p}=0.006\right]$ and very likely $(0.45$; $234 \pm 0.35)$ greater $\Delta \mathrm{Tc}$ in game 1 compared to game $3\left[0.30\left(0\right.\right.$ to $\left.\left.0.61^{\circ} \mathrm{C}\right) ; \mathrm{p}=0.05\right]$. No differences 235 for any other Tc derived measure was evident between games $(p \geq 0.27)$ or between WU ( $p \geq$ $2360.53)$.

237 Effects of minutes played and external load (GPS) variables on peak Tc (all players): Minutes 238 played was associated with peak Tc $(\mathrm{p}=0.04)$. Minutes played very likely $(0.71 ; \pm 0.28)$ resulted 239 in an increased peak Tc. There was no association between any of the external load (GPS) $240 \quad$ variables and peak $\mathrm{Tc}(\mathrm{p} \geq 0.22)$.

241 Effects of minutes played and external load (GPS) variables on peak Tc (only players $\geq 6$ min):

242 No association $(\mathrm{p}=0.11)$ between minutes played and peak Tc. There was no association 243 between any of the external load (GPS) variables and peak Tc ( $\mathrm{p} \geq 0.34)$.

244 External load $(G P S)$ data (only players $\geq 6 \mathrm{~min})$ : Differences in average speed $\left(\mathrm{m} \cdot \mathrm{min}^{-1}\right)(\mathrm{f}=$ 245 6.81, $\mathrm{p}=0.009), \mathrm{HSR} \cdot \mathrm{min}^{-1}(\mathrm{f}=10.74, \mathrm{p}=0.002), \operatorname{VHSR} \cdot \mathrm{min}^{-1}(\mathrm{f}=65.86, \mathrm{p}<0.001)$, and 246 Ave Acc/Dec $\left(\mathrm{m} \cdot \mathrm{s}^{-2}\right)(\mathrm{f}=6.17, \mathrm{p}=0.007)$ were evident (see Table 5 for specifics). 
*** Table 5 near here please $* * *$

248 Cooling intervention use (only players $\geq 6 \mathrm{~min}$ ): For the first CWI players had different 249 exposure times [6 (4-6 min)]. The second and third exposure was standardised to 5 and $6 \mathrm{~min}$ 250 respectively. Differences were not seen for $\Delta \Delta \mathrm{CWI}(\mathrm{p}=0.10)$, see Figure 3. Player cooling 251 rates were calculated by dividing the $\Delta \Delta \mathrm{CWI}$ by CWI duration $\left(\Delta \Delta \mathrm{CWI}{ }^{\circ} \mathrm{C} \cdot \mathrm{min}^{-1}\right)$, and then by 252 lean body mass (LBM; $\left.\Delta \Delta \mathrm{CWI}{ }^{\circ} \mathrm{C} \cdot \mathrm{min}^{-1} 1 . \mathrm{LBM}\right)$, see Figure 3.

*** Figure 3 near here please $* * *$

254 Perceptual data (only players $\geq 6 \mathrm{~min}$ ): Differences $(\mathrm{p}=0.001)$ in TC between games were 255 evident (see Table 3$)$. Players most likely $(-3.2 ; \pm 0.57)$ felt more 'comfortable' with the 256 temperature of their body in game 3 compared to game 1 [-1.06 $(-0.6$ to -1.6$) ; p=0.001]$, and game $2[-1.04(-0.5$ to -1.5$) ;-4.0 ; \pm 1.20 ; \mathrm{p}=0.001]$. No differences were evident regarding RPE $(p=0.30)$ or TS $(p=0.12)$, see Figure 4.

*** Figure 4 near here please $* * *$

EHI signs and symptoms: no signs and symptoms of EHI were reported by any player.

\section{Discussion}

262 Approximately $50 \%$ of players demonstrated a peak Tc $\geq 39^{\circ} \mathrm{C}$ in response to WRWSS match263 play within mild WBGT, a magnitude proposed to decrease high-intensity intermittent physical 264 performance ${ }^{4}$; accepting experimental hypothesis (i) and in line with male player data. ${ }^{3}$ 265 Additionally, the employed CWI protocol left players ( $\geq 6$ min match-play) with a $\sim 1-2^{\circ} \mathrm{C}$ 266 raised Tc, compared to Tc at WU onset (Figure 3) in acceptance of hypothesis (ii). Together 267 this demonstrates that despite brief match-play duration within modest WBGT environments, 268 athletes can experience a Tc rise that decreases performance ${ }^{4}$; whilst the CWI protocol 
employed fails to acquiesce the majority of the acquired Tc rise (e.g. transient hyperthermia) during each game.

271 Despite modest/low WBGT $\left(18.5-20.1^{\circ} \mathrm{C}\right)$, high peak Tc values $\left(37.9-39.8^{\circ} \mathrm{C} ; \sim 50 \% \geq\right.$ $27239^{\circ} \mathrm{C}$ ) were observed (particularly in those with $\geq 6$ min match-play) reaching magnitudes 273 known to reduce repeat sprint ability. ${ }^{4}$ Research from other sports sharing high-intensity, intermittent physical demands (soccer and tennis) has reported elite females recording similar peak Tc values $\left(38-40^{\circ} \mathrm{C}\right){ }^{23,24}$ Given the relationship [very likely $(0.71 ; \pm 0.28)$ ] seen between minutes played and peak Tc, practitioners and coaches seeking to control player Tc and maximise performance may benefit from pre or per-cooling interventions in the short term or increasing heat tolerance in the longer term. Long-term heat acclimation strategies, although not always practically compatible with training/competition, ${ }^{25}$ have been shown to provide the greatest protection to performance decrements and EHI. ${ }^{26}$

Alternatively, opting for an alternate WU environment with more controlled conditions (e.g. indoor) or modifying the WU volume/duration/intensity and thus reducing body heat storage from it, may provide a greater Tc reserve until $\mathrm{a} \geq 39^{\circ} \mathrm{C}$ is reached during match-play (i.e. players start the match-cooler). Indeed, body heat storage from the employed WU could be considered 'too much' (Figure 2) given a peak Tc of $39^{\circ} \mathrm{C}$ was seen for two players and exceeding $38.5^{\circ} \mathrm{C}$ in $>50 \%$ of players. Practically however, such titration of WU may be

287 challenging to achieve, given often divergent player needs and the dynamic nature of individual

288 Tc change across the day. There are obvious practical and logistical advantages in allowing all squad members to complete the same WU, as a team, during a WRWSS match-day. This may be possible if augmented heat loss was facilitated during the WU, which through the use of a

291 phase change cooling vest has shown promise as an effective and ecologically valid strategy to 292 reduce Tc, without altering selected WU characteristics or performance measures in elite men's 
293 rugby sevens. ${ }^{12}$ However, specific to the WRWSS there is currently no evidence regarding the

294 efficacy of such interventions, which may be different to those presented for elite male sevens

295 players $^{12}$ (for reasons outlined in the introduction). Further, the Tc responses discussed above

296 from modest WBGT environments, would likely be exacerbated in high WBGT environments,

297 such as those experienced commonly in other WRWSS tournaments and expected at Tokyo

$2982020 .^{6,7}$ During or proximal to the warm-up, strategies to minimise hyperthermia risk and/or

299 increase the Tc heat-sink (and associated favourable perceptual changes) prior to competition,

300 appear prudent. These include, amongst others, wearing a phase change cooling vest, ice-slurry

301 ingestion after the sweat response has been observed, ice towel application, performing the

302 warm-up in an air-conditioned indoor environment (with simulated wind velocity), or outdoors

303 with appropriate wind velocity (N.B. such approaches require extensive piloting and should

304 not compromise the desired outcome of an effective warm-up).

305 Quickly removing the majority of the rise in Tc due to match-play appears prudent, given the

306 accumulation of Tc between games, despite similar work rates across games. Indeed, average

307 Tc values in the present data were most likely higher in game 2 than game 1, and very likely

308 higher in game 3 than game 2 (players with $\geq 6$ minutes involvement time), in support of similar

309 findings in elite men's rugby sevens who did not perform any in-competition cooling. ${ }^{3}$

310 However, the employed CWI protocol left players ( $\geq 6$ min match-play) with $\mathrm{a} \sim 1-2^{\circ} \mathrm{C}$ raised

311 Tc compared to Tc at WU onset (Figure 3). Evidently, the lack of individualisation of CWI

312 time (e.g. by match-play minutes) and that players only immersed themselves to hip height,

313 contributed to the cooling responses seen (despite the CWI water temperature range 6.1 -

$3147.3^{\circ} \mathrm{C}$ ). Piloting of both these CWI protocol modifications [e.g. individualised immersion based

315 on minutes played and shoulder-depth immersion (to maximise conductive heat dissipation ${ }^{27}$ )]

316 is recommended, to produce a practice ready individualised solution. 
317 Although previous research has indicated that high Tc values $\left(>39^{\circ} \mathrm{C}\right)$ are detrimental to repeat 318 and intermittent sprint performance, ${ }^{4}$ the present study found no link between changes in Tc

319 and reductions in external load measures (GPS). This could be due to individual variation in

320 the relationship between high Tc and repeat and intermittent sprint performance as discussed

321 elsewhere, ${ }^{4}$ or technical/tactical contextual factors of the matches influencing their physical

322 demands, ${ }^{28}$ or a sample size $(\mathrm{n}=12 ; 3$ matches $)$ too small to detect a meaningful change in external load (GPS) metrics. ${ }^{29}$ A lack of robust data regarding the relationship between altered thermal perceptions and their effect on performance capacities in elite athletes (e.g. particularly repeat sprint ability), renders performance-based inferences on perceptual measures uncertain within the present design; although reductions in thermal sensation without accompanying physical body temperature decreases can within some scenarios (predominately endurance exercise) prove ergogenic to exercise performance in the heat. ${ }^{12,30}$ Future research should look to include multiple match-days and/or tournaments so contextual variance due to technical/tactical factors is less pronounced, and greater statistical power can be attained.

\section{Practical Applications}

- Modifying the WU volume/duration/intensity to reduce body heat storage may provide

- Individualised CWI protocols based on minutes played and shoulder-depth immersion is recommended to produce a practice-ready individualised solution to heat dissipation.

\section{Conclusions}

338 Like their male counterparts, elite female rugby sevens athletes experience high Tc during WU

339 and matches on WRWSS match-days, even in modest WBGT environments. Strategies and 
modifications to match-day protocols likely to result in improved thermoregulatory function, and subsequent physical performance for athletes are available, yet they lack empirical data to inform practice with specificity to the WRWSS.

\section{Declarations/Acknowledgements}

344 The authors thank the players and staff for their willingness, professionalism, engagement and time pre, during and post the executed research project - without which - the project would not have been viable. Lee Taylor (and co-authors) thanks the Aspire Zone Foundation (AZF; Doha, Qatar) for the funding and support to complete the presented research project. Additional thanks go to BodyCap (Caen, France) for the loan of 12 e-Viewers (plus general technical and logistical support) to complete the presented research project. Mitchell J. Henderson was supported by the RTP scholarship (Australia) and a research scholarship from Rugby Australia for this project. Aside from the above there was no further potential conflict of interests for any author in this study. Each author contributed to experimental design, data collection and data analysis, manuscript drafting and agreed to the submitted version of the manuscript.

\section{References}

1. Ross A, Gill N, Cronin J. The match demands of international rugby sevens. $J$ Sports Sci. 2015;33(10):1035-1041.

2. Clarke AC, Anson JM, Pyne DB. Game movement demands and physical profiles of junior, senior and elite male and female rugby sevens players. J Sports Sci. 2017;35(8):727-733.

3. Taylor L, Thornton HR, Lumley N, Stevens CJ. Alterations in core temperature during World Rugby Sevens Series tournaments in temperate and warm environments. Eur J Sport Sci. 2019;19(4):432-441.

4. Girard O, Brocherie F, Bishop DJ. Sprint performance under heat stress: A review. Scand J Med Sci Sports. 2015;25 Suppl 1:79-89.

5. Kaciuba-Uscilko H, Grucza R. Gender differences in thermoregulation. Curr Opin Clin Nutr Metab Care. 2001;4(6):533-536.

6. Kakamu T, Wada K, Smith DR, Endo S, Fukushima T. Preventing heat illness in the anticipated hot climate of the Tokyo 2020 Summer Olympic Games. Environ Health Prev Med. 2017;22(1):68.

7. Kashimura O, Minami K, Hoshi A. Prediction of WBGT for the Tokyo 2020 Olympic Marathon. Japanese Journal of Biometeorology. 2016;53(4):139-144. 
8. Janse DEJXA, Thompson MW, Chuter VH, Silk LN, Thom JM. Exercise performance over the menstrual cycle in temperate and hot, humid conditions. Med Sci Sports Exerc. 2012;44(11):2190-2198.

9. Ihsan M, Watson $\mathrm{G}$, Abbiss CR. What are the physiological mechanisms for postexercise cold water immersion in the recovery from prolonged endurance and intermittent exercise? Sports Med. 2016;46(8):1095-1109.

10. Schuster J, Howells D, Robineau J, et al. Physical-preparation recommendations for elite rugby sevens performance. Int J Sports Physiol Perform. 2018;13(3):255-267.

11. Bongers C, Daanen HAM, Bogerd CP, Hopman MTE, Eijsvogels TMH. Validity, reliability, and inertia of four different temperature capsule systems. Med Sci Sports Exerc. 2018;50(1):169-175.

12. Taylor L, Stevens CJ, Thornton HR, Poulos N, Chrismas BCR. Limiting the rise in core temperature during a rugby sevens warm-up with an ice vest. Int $J$ Sports Physiol Perform. 2019:1-20.

13. Byrne C, Lim CL. The ingestible telemetric body core temperature sensor: a review of validity and exercise applications. Br J Sports Med. 2007;41(3):126-133.

14. Travers GJS, Nichols DS, Farooq A, Racinais S, Périard JD. Validation of an ingestible temperature data logging and telemetry system during exercise in the heat. Temperature. 2016;3(2):208-219.

15. Periard JD, Racinais S, Timpka T, et al. Strategies and factors associated with preparing for competing in the heat: a cohort study at the 2015 IAAF World Athletics Championships. Br J Sports Med. 2017;51(4):264-270.

16. Thornton HR, Nelson AR, Delaney JA, Serpiello FR, Duthie GM. Interunit reliability and effect of data-processing methods of Global Positioning Systems. Int J Sports Physiol Perform. 2019;14(4):432-438.

17. Young AJ, Sawka MN, Epstein Y, Decristofano B, Pandolf KB. Cooling different body surfaces during upper and lower body exercise. J Appl Physiol. 1987;63(3):1218-1223.

18. Borg GA. Psychophysical bases of perceived exertion. Med Sci Sports Exerc. 1982;14(5):377-381.

19. Hopkins WG, Marshall SW, Batterham AM, Hanin J. Progressive statistics for studies in sports medicine and exercise science. Med Sci Sports Exerc. 2009;41(1):3-13.

20. Grafen G, Hails R. In: Modern Statistics for the Life Sciences. New York, USA: Oxford University Press; 2002:153 - 184

21. West BT, Welch KB, Galecki AT. Linear Mixed Models: A Practical Guide Using Statistical Software, Second Edition. Taylor \& Francis; 2014.

22. Hurvich CM, Tsai CL. Model selection for extended quasi-likelihood models in small samples. Biometrics. 1995;51(3):1077-1084.

23. Tippet ML, Stofan JR, Lacambra M, Horswill CA. Core temperature and sweat responses in professional women's tennis players during tournament play in the heat. $J$ Athl Train. 2011;46(1):55-60.

24. Somboonwong J, Chutimakul L, Sanguanrungsirikul S. Core temperature changes and sprint performance of elite female soccer players after a 15-minute warm-up in a hothumid environment. J Strength Cond Res. 2015;29(1):262-269.

25. Casadio JR, Kilding AE, Cotter JD, Laursen PB. From Lab to Real World: Heat Acclimation Considerations for Elite Athletes. Sports Med. 2017;47(8):1467-1476.

26. Racinais S, Alonso JM, Coutts AJ, et al. Consensus recommendations on training and competing in the heat. Br J Sports Med. 2015;49(18):1164-1173.

27. Zhang Y, Davis JK, Casa DJ, Bishop PA. Optimizing cold water immersion for exercise-induced hyperthermia: a meta-analysis. Med Sci Sports Exerc. 2015;47(11):2464-2472. 
28. Henderson MJ, Harries SK, Poulos N, Fransen J, Coutts AJ. Rugby sevens match demands and measurement of performance: a review. Kinesiology. 2018;50:49-59.

29. Biau DJ, Kernéis S, Porcher R. Statistics in brief: the importance of sample size in the planning and interpretation of medical research. Clin Orthop Relat Res. 2008;466(9):2282-2288.

30. Stevens CJ, Mauger AR, Hassmen P, Taylor L. Endurance Performance is Influenced by Perceptions of Pain and Temperature: Theory, Applications and Safety Considerations. Sports Med. 2018;48(3):525-537.

\section{Figure captions}

Figure 1. Experimental schematic. Tc = core temperature (ingestible telemetric pill); GPS = Global Positioning Systems; TS $=$ thermal sensation; $\mathrm{TC}=$ thermal comfort; $\mathrm{RPE}=$ rating of perceived exertion; CWI = cold water immersion; $\mathrm{WU}=$ warm-up.

Figure 2. Individual average (A), peak (B), $\Delta$ period (C) and $\Delta$ baseline (D) core temperature (Tc) responses across all periods of the tournament for all players. The solid black line represents the mean for all players. The dashed line represents $39^{\circ} \mathrm{C}$. Open circles represent players who had $\geq 6$ min match-play. The likelihood of the observed effect exceeding the smallest worthwhile change $(0.2 \mathrm{x}$ between subject $\mathrm{SD})$ when compared to baseline are denoted as; $* *=$ very likely, $* * *=$ most likely. When compared to game 1 differences are expressed as; $\dagger \dagger=$ very likely. When compared to game 1 only for players with $\geq 6$ min match-play, differences are expressed as ${ }^{\wedge \wedge}=$ very likely, and ${ }^{\wedge \wedge}=$ most likely. $\mathrm{BL}=$ baseline; $\mathrm{WU}=$ warmup; $\mathrm{G}=$ game; $\mathrm{CWI}=$ cold water immersion.

Figure 3. Individual $\Delta$ combined (A), $\Delta \Delta \mathrm{CWI}(\mathrm{B})$, cooling rate $\left({ }^{\circ} \mathrm{C} \cdot \mathrm{min}^{-1}\right)(\mathrm{C})$ and relative cooling rate $\left({ }^{\circ} \mathrm{C} . \mathrm{min}^{-1}\right.$.LBM) (D) core temperature $(\mathrm{Tc})$ responses to cold water immersion (CWI) and games respectively, for all players. The solid black line represents the mean. Open circles represent players who had $\geq 6$ min match-play.

Figure 4. Individual RPE (A), TC (B) and TS (C) responses to games 1, 2 and 3 for all players. The solid black line represents the mean. Open circles represent players who had $\geq 6 \mathrm{~min}$ match-play. The likelihood of the observed effect exceeding the smallest worthwhile change $(0.2 \mathrm{x}$ between subject SD) when compared to game 1 are denoted as; $* * *=$ most likely. When compared to game 2 differences are expressed as; $\dagger \dagger \dagger=$ most likely. $\mathrm{RPE}=$ rating of perceived exertion; $\mathrm{TC}=$ thermal comfort; $\mathrm{TS}=$ thermal sensation. 


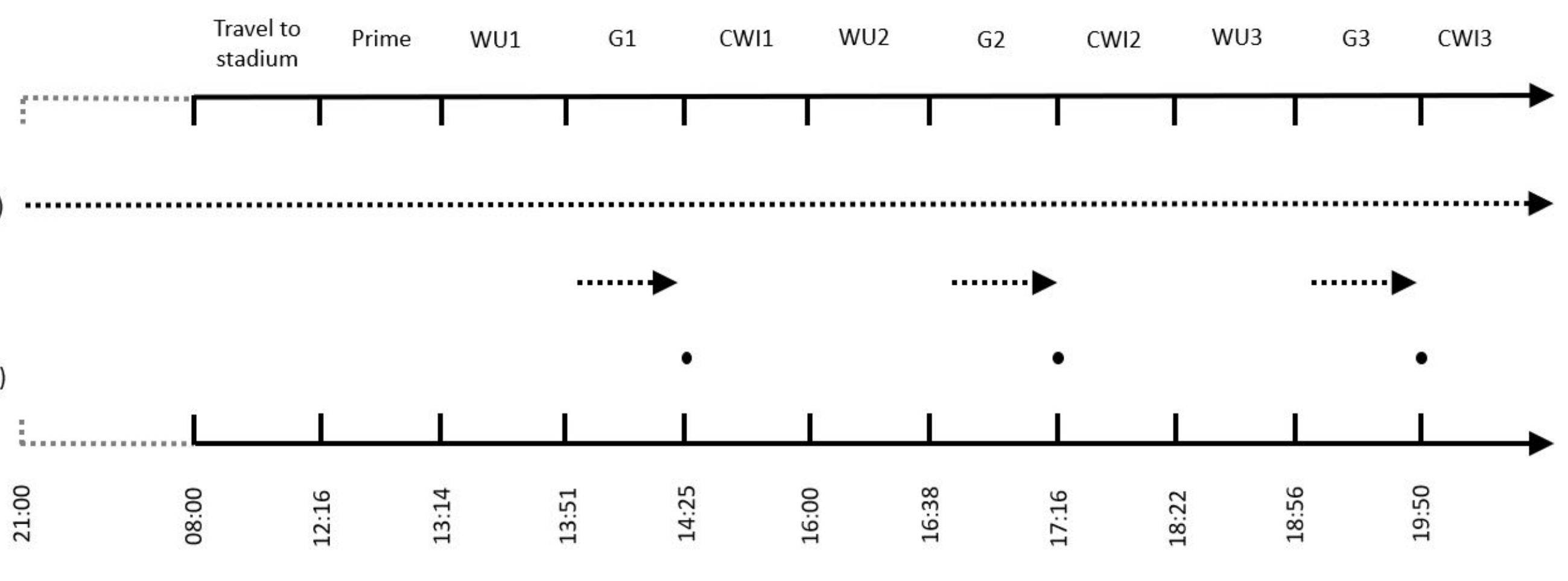





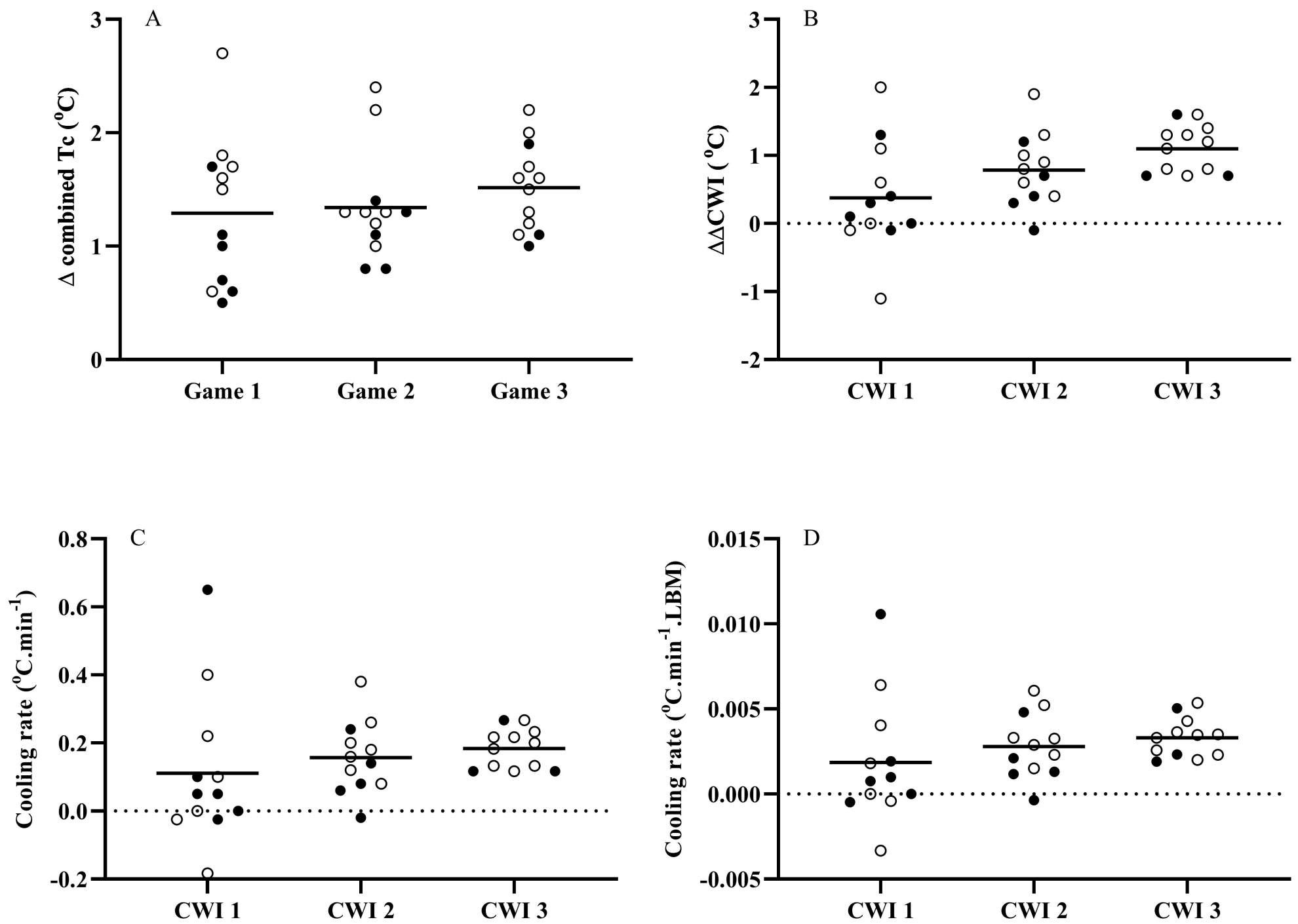

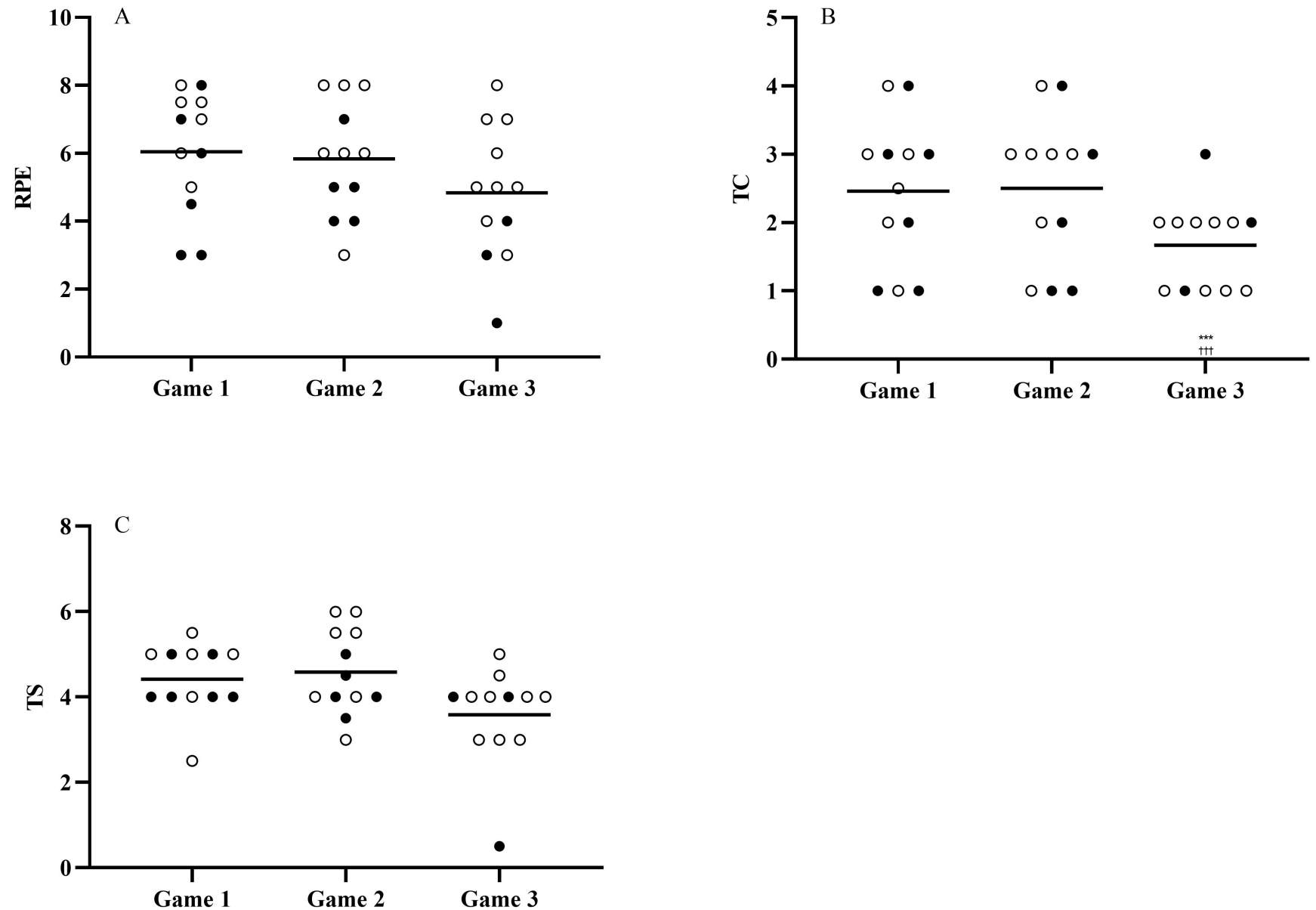




